\title{
Cartographic modeling for monitoring the technical condition of urban development objects
}

\author{
Marina Dement'eva ${ }^{1, *}$, and Anastasiya Dement'eva ${ }^{1}$ \\ ${ }^{1}$ Moscow State University of Civil Engineering, Yaroslavskoe shosse, 26, Moscow, 129337, Russia
}

\begin{abstract}
The paper presents the results of a study of the risk factors for the degradation of the urban ecosystem and their impact on the state of the objects of urban development. The purpose of the study was the development of a cartographic model for predicting the change in the technical condition of a town-planning facility, taking into account external factors. The relevance of the work is justified by the increase in the flow of failures of constructions and building systems, as well as the absence of a single system for diagnosing the risk of emergency situations. In the course of the study, the methods of mapping, structural analysis, the index method, and the method of expert assessments were used. The criterion for estimating the degree of influence of the operating environment on the exploited objects, which takes into account the quality of the medium and the decrease in the durability of the object, is proposed in this work. An example of the development of a forecast scale for the operating conditions of an object in the form of a matrix model is given in this paper. On the basis of the developed cartographic model, the cause-effect relationships between the intensity of failures on the exploited objects and the degree of degradation of the city's ecosystem were revealed. The model proposed by the authors can be the basis for adjusting the monitoring of technical condition, methods of protecting structures from destruction and optimizing the planning of preventive repairs.
\end{abstract}

\section{Introduction}

Modern cities are an artificially created habitat, which is designed to meet all human needs. The city should be provided with the best conditions for life and human activity, for the implementation of various functional processes. The concept of sustainable development of the city assumes that for this to be ensured the safety and comfort of the city as an object of use. However, a characteristic and large-scale problem of modern cities are man-caused impacts. The imbalance of territories of natural and anthropogenic origin led not only to the disastrous impact on the state of the components of the natural ecosystem of the city, but also negatively affected the components of anthropogenic systems. Numerous studies on the state of the urban ecosystem show [1-6] that in the last decade the environment of

\footnotetext{
*Corresponding author: 7dem@mail.ru
} 
exploitation has changed. In this case, damage to the ecosystem in places of extreme stress on nature is accompanied by emergency situations in the urban economy. Frequent accidents lead to significant material costs, loss of resources, entail a deterioration of the environmental situation, a decrease in safety and quality of life. Therefore, it is necessary to take this factor into account when solving critical problems at the objects of urban development.

Obviously, in order to ensure the safety and reliable exploitation of construction projects in such conditions, it is necessary to define fundamentally new ecological approaches to the development of a system for forecasting damage risks. This will help to establish the priority of preventive measures to protect against damage to specific objects located in areas of extreme loads from the urban environment. At the same time, it is often necessary to observe that the section of the system of preventive measures is poorly justified in the technological scheme of design works. But it is these technologies that are called to ensure the sanitation and protection of building constructions and engineering systems from external influences. The lack of this system of measures leads to the fact that soon after repair or reconstruction, corrective measures must be actively used. There are additional financial and material costs.

The hypothesis of the study was that one of the reasons for the problem in question is the lack of a well-organized system for rapid examination and systematization of the information that has been collected and already accumulated, for example, chemical loads of the environment on the structure, causes and types of damage. Urgency in the implementation of measures to eliminate and prevent accidents requires new technologies in the scientific and methodological plan for solving practical problems in emergency situations. It has become unacceptable to conduct long-term studies. Information systems of already available scientific knowledge and experience are needed, which will quickly help to find the necessary answers in such situations [7-9].

As a solution to the problem in question, it was suggested to use cartographic modeling for monitoring the technical condition of urban development objects. A review of literature and research shows [10-13] that the use of cartographic models with sufficient detail of natural and man-made components can serve as a basis for effective forecasting, both technogenic changes in the environment and the risk of emergencies. However, most of the research is devoted to the digitization of traditional maps [14, 15], the development of methods for improving the data of topographic, geodetic, ecological and other maps [16, 17]. There are also working software packages for the development of map-case of emergency services of engineering systems [18], which allow to fix the failure rate and establish its relationship with internal factors, for example, the service life, the materials used, the frequency of repairs, etc. At the same time, there is no systematic fixation of data on the state of structural elements of the city buildings. Therefore, for a more complete analysis, data on external influences are needed, which justifies the relevance of this work.

The most promising are the so-called synthetic maps [19], with the help of which, in the opinion of the authors, the following problems can be solved:

- establish correlation links between environmental pollution components and their sources;

- to identify the ways of the most rational reduction of the impact of environmental components on constructions and buildings systems;

- provide for measures to prevent the development of destructive processes in constructions and buildings systems;

- determine the conditions under which the environment of exploitation, which experiences an external technogenic impact, will maintain a stable state, will continue to perform socio-economic, engineering and technical functions within specified limits. 
Therefore, the purpose of this study was to develop a cartographic model for predicting the change in the technical state of a town-planning facility, taking into account external factors. To achieve it, you need to solve the following tasks:

- develop an algorithm for the formation of a cartographic model;

- analyze the combination of man-made factors and assess their relationship to the risk of emergency situations;

- propose a forecast scale for changing conditions of exploitation.

\section{Materials and Methods}

The object of the study was the process of developing a cartographic model for finding the correlation dependence between the parameters conditions of exploitation and changing the characteristics of the exploited object. The subject of the study was the study of techniques for the formation of cartographic models that contribute to improving the monitoring of the technical condition of urban development facilities. In the development of cartograms, the methods of mapping, structural analysis, the index method, the method of expert assessments were used.

Mapping was performed on the example of the city of Moscow. The territory of Moscow was divided into map-case - squares $0.5 \times 0.5 \mathrm{~km}$ in scale. Further, by the method of system analysis and spatial variation, a cartogram of the total level of the projected load in the city territory and the risk of damage to constructions, engineering systems based on environmental characteristics was obtained.

As statistical materials used in the work for sampling and systematization of data on the load on the territory, the basis is taken [20-23]:

- map of groundwater pollution (Figure 1a);

- map of landfill location in the city (Figure 1b);

- map of location of industrial and communal-warehouse zones and enterprises (Figure $1 \mathrm{c})$;

- map of the change in the corrosion activity of soils in terms of the specific electrical resistance (Figure 1d);

- map of the location of natural complexes in the city (Figure 1e).

To systematize the data on the technical condition, a map of damage to underground water pipes was selected (Figure 1f), based on the following considerations:

- the availability of systematically fixed information on the flow of failures, in contrast to the constructions of buildings for which such information is not available;

- the possibility of fixing information about the technical state both directly and indirectly (pressure drop, leakage, water quality decrease, etc.);

- the stability of the internal environment in comparison with in hot water supply, in the heat network allows to level out the impact of internal factors when assessing the causes of damage.

In each square, all the loads presented above were summed up. It was suggested that such a collection of data takes into account both negative man-made factors and positive natural factors that have the most significant impact on the environment of exploitation. In particular, natural complexes contribute to reducing the risk of degradation of the soil ecosystem, compensate for air emissions, etc. This justifies the likelihood of a decrease in the activity of the environment of exploitation with respect to the monitoring facility.

In the analysis of the ecological situation and the synthesis of information, the quantitative and qualitative environmental characteristics of the urban environment and the principle of the probability of their territorial manifestation were taken into account. To be able to take into account all types of loads, regardless of their range and dimensionality, it was necessary to bring them into a single coordinate system. 

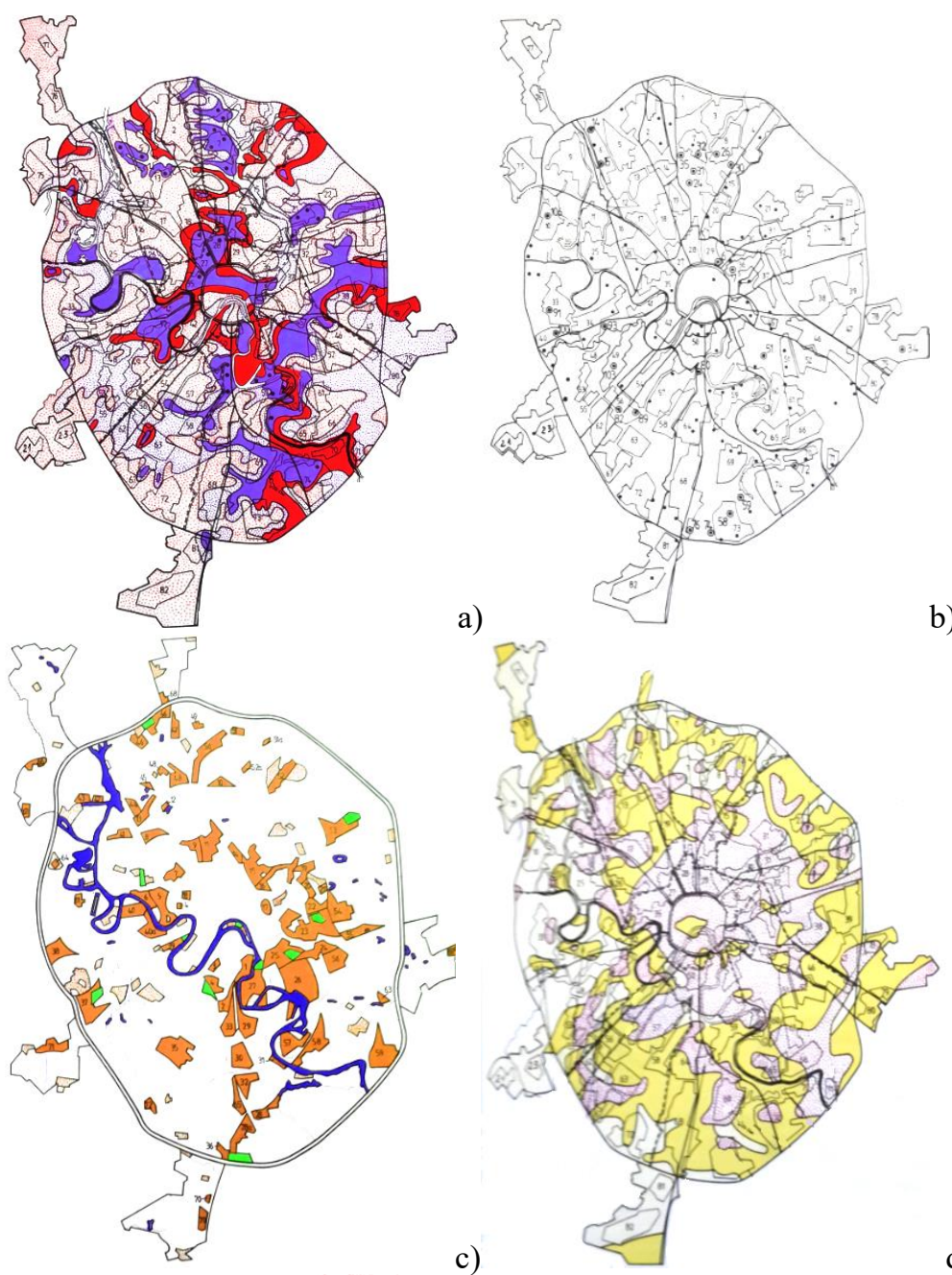

b)
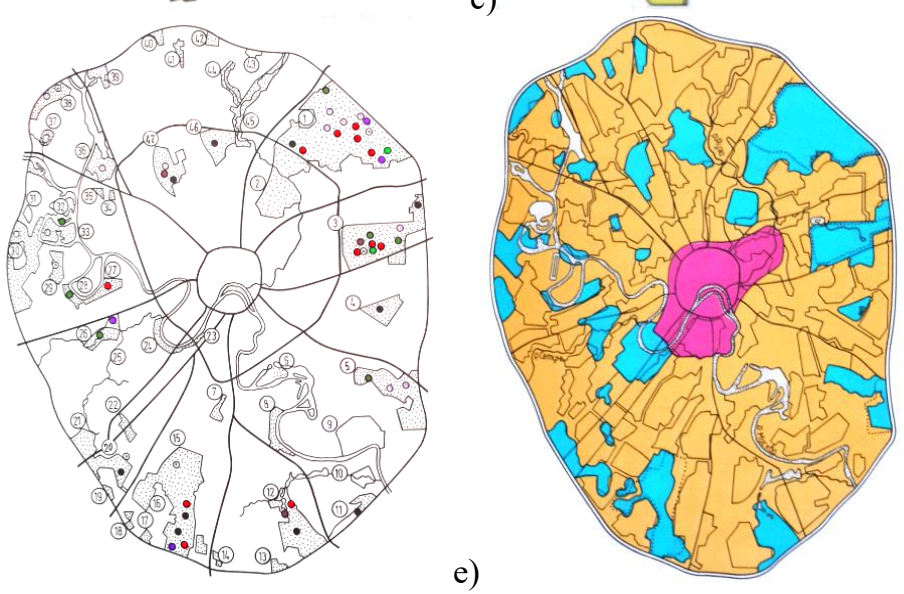

d)

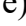

f)

Fig. 1. Map of the city of Moscow: a) map of groundwater pollution; b) map of the location of landfills; c) map of the location of industrial and communal enterprises; d) map of soil corrosion activity; e) map of natural complexes; f) map of leaks from water-bearing communications. 
For this purpose, it is proposed to calculate the index of the quality of the environment based on the index method on the basis of the value of the load:

$$
k_{i}=\frac{\chi_{i}^{f}-\chi_{i}^{\min }}{\chi_{i}^{\max }-\chi_{i}^{\min }}
$$

where $k_{i}$ is the index of the influence of the $\mathrm{i}$-th load on the environment of exploitation; $\chi_{i}^{f}$ is the actual value of the i-th load, determined by the results of monitoring the characteristics of the operating environment; $\chi_{i}^{\text {min }}$ is the minimum value of the $\mathrm{i}$-th load; $\chi_{i}{ }^{\max }$ is the maximum value of the i-th load.

The index $k_{i}$ characterizes the degree of approximation of the actual load value to the maximum and minimum values and is an absolute indicator of the quality of the environment of exploitation.

At the same time, it is necessary to assess the degree of influence of a specific load on the object of urban development. As the evaluation criteria, it was proposed reliability indicators, for example, the probability of failure or change in the durability of the object. The first indicator is advisable to apply for engineering systems, since the failure flow is relatively high and technically easy to register. The second indicator is more expedient to apply for more durable objects, for example, constructions of building. The degree of influence of the load depends on many factors: the prevalence of the impact, its effect on the concrete building material, the type of damage caused, the effect of damage on the functioning of the facility, and the quality of the urban environment, etc. To evaluate the degree of influence of the load on the object and the summation of the types of impacts under consideration, the authors suggested using mathematical procedures based on the method of expert assessments. The significance of the load $r$ is proposed to be evaluated depending on the value of the deviation of the actual time between failures $T_{f}$ from the normative life $T_{n}$ (Table 1):

$$
\Delta t=100 \frac{T_{n}-T_{f}}{T_{n}}
$$

Table 1. Expert evaluation of the degree of influence of anthropogenic load on the object.

\begin{tabular}{|c|c|c|}
\hline Degree of influence & The amount of deviation $\Delta \boldsymbol{t}$ & Importance of load $\boldsymbol{r}$ \\
\hline Weak S1 & $\Delta t \leq 0$ & 0.1 \\
\hline Medium S2 & $0<\Delta t \leq 20$ & $0.2-0.3$ \\
\hline Strong S3 & $20<\Delta t \leq 50$ & $0.4-0.6$ \\
\hline Maximum S4 & $50<\Delta t \leq 70$ & $0.7-0.8$ \\
\hline Extreme S5 & $\Delta t>70$ & $0.9-1.0$ \\
\hline
\end{tabular}

The total influence index $I_{d}$ of the aggregate of loads on the durability of the object of town-planning activity, on the probability of its failure before the expiration of its service life, it is proposed to determine by the formula:

$$
I_{d}=\sum_{i=1}^{n} k_{i} \cdot r_{i}
$$




\section{Results}

To refine the data during the formation of the cartographic model, an algorithm for analyzing destructive processes at the objects of the city infrastructure was developed (Figure 2). This algorithm is intended not only for diagnosis and rapid analysis of the technical condition of the object. The ultimate goal is to develop a system of corrective organizational and technological operations to restore the operability of the facility and to prevent subsequent intensive wear and tear.

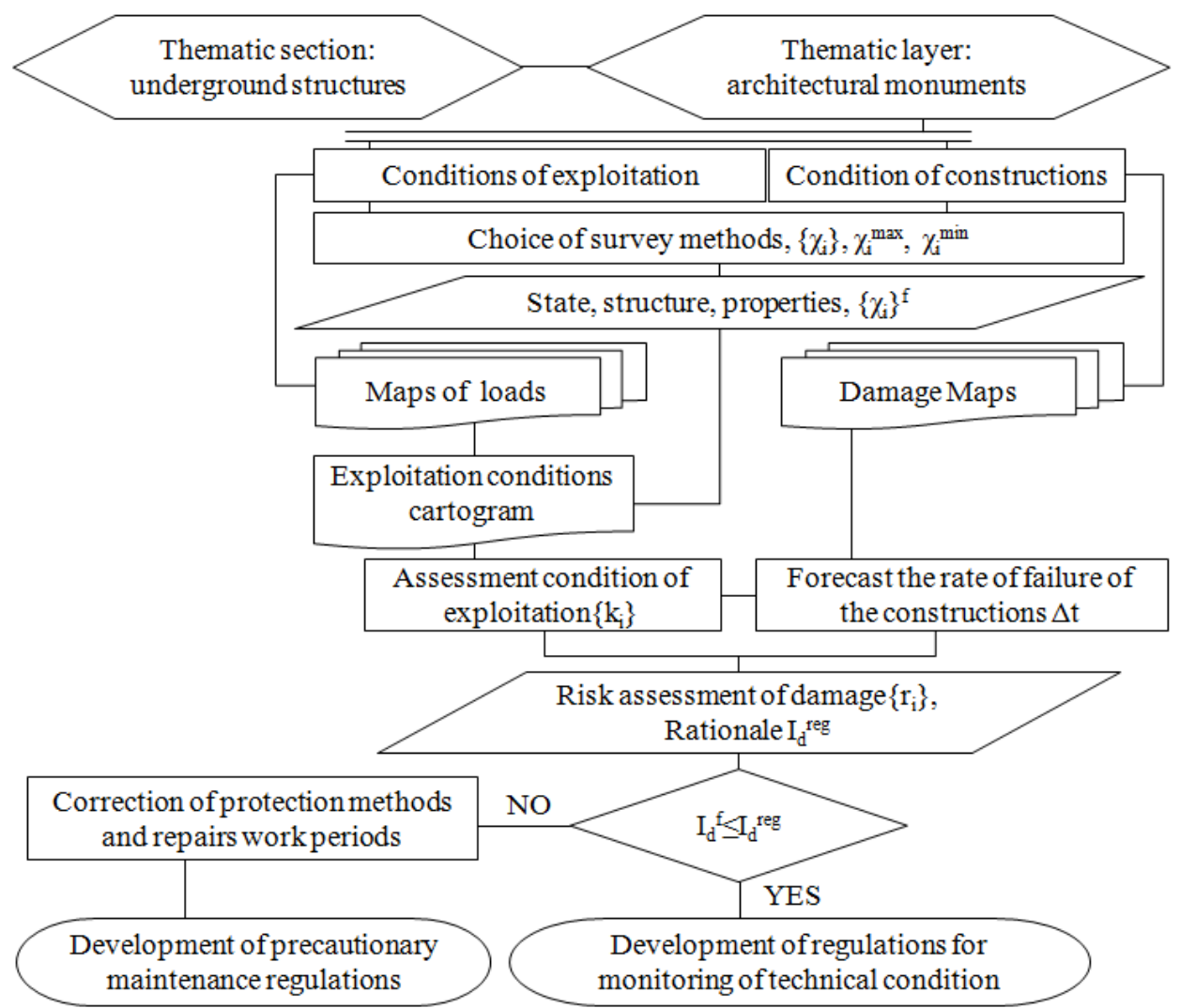

Fig. 2. Algorithm for forming a cartogram of the forecast load for monitoring destructive processes on the example of underground urban development objects.

Based on the developed algorithm, a cartogram of total loads was constructed (Figure 3 ), combinations of technogenic factors were analyzed and their interrelation with the risk of emergencies was studied using the example of a cold water supply system in the city of Moscow. A layered analysis of the information confirmed the existence of correlation links between the magnitude of the loads and the destructive processes in the objects. This confirms the validity of the proposed methodology for assessing the technical condition of the objects of urban development. In places with the highest influence index $I_{d}$, private pipeline failures are indeed recorded.

To calculate the total impact index $I_{d}$ and the completeness of accounting for all factors of the negative impact on the object, a forecast scale for the conditions of exploitation was compiled (Table 2), which was used in the development of the cartogram in Figure 3. The influence of five types of loads was taken into account:

$\mathrm{S} 1$ - degree of groundwater pollution (index of quality of environment $k 1$, importance of load $r l$ ); 


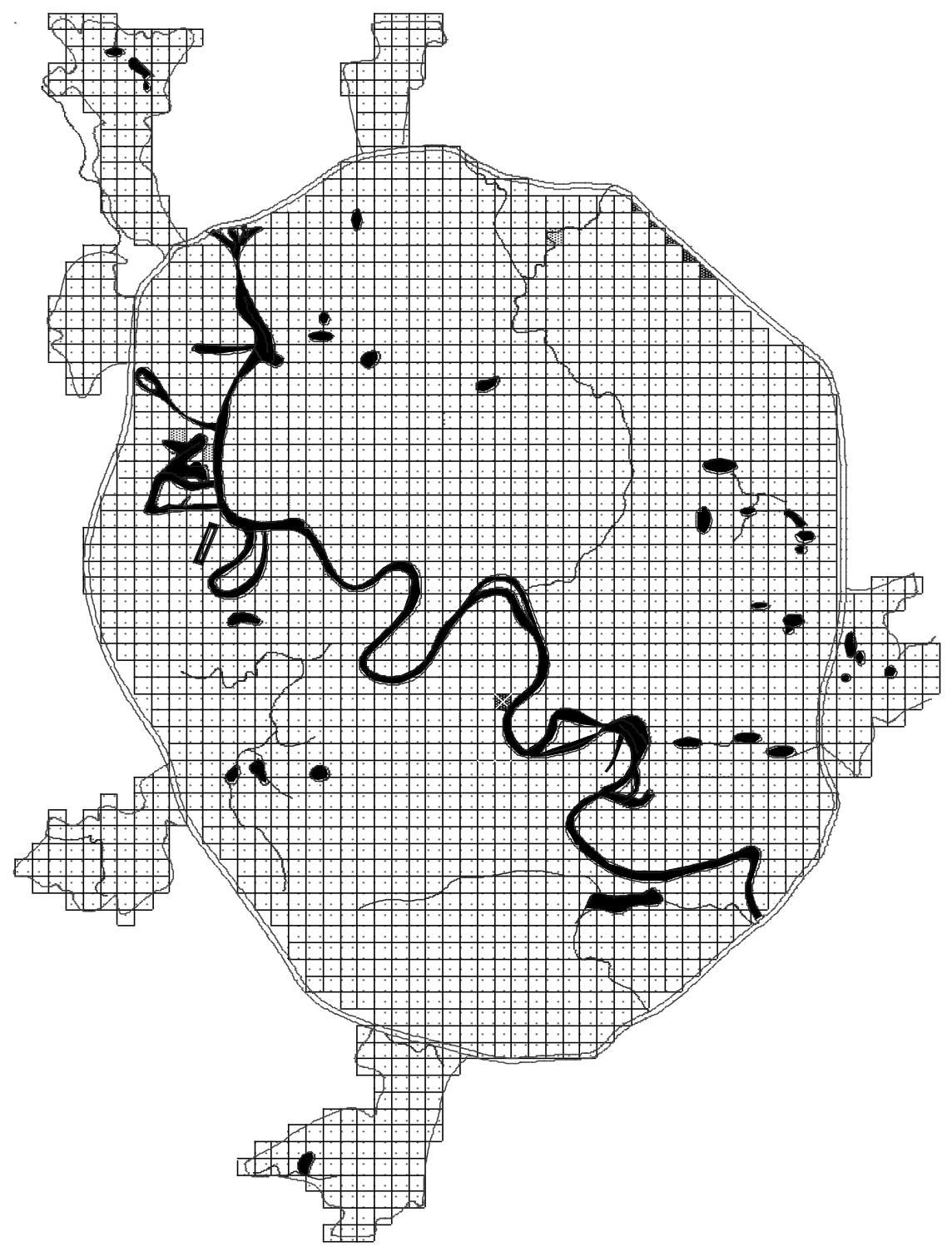

Fig. 3. Cartogram of the total load on underground objects of town-planning activity.

$\mathrm{S} 2$ - presence of industrial zones (index of quality of environment $k 2$, importance of load $r 2$ );

$\mathrm{S} 3$ - the presence of corrosive soils (index of quality of environment $k 3$, importance of load $r 3)$;

S4 - availability of landfills (index of quality of environment $k 4$, importance of load $r 4)$;

S5 - the presence of natural communities (index of quality of environment $k 5$, importance of load $r 5$ ).

It must be remembered that the fifth load is positive from the point of view of influence both on the environment of exploitation and on the object, therefore it is taken into account with the opposite sign. The second, fourth and fifth loads have an indirect effect on the magnitude of the first and third, so they are estimated on a binary scale. 
Table 2. Matrix for the development of the forecast scale conditions of exploitation of the facility.

\begin{tabular}{|c|c|c|c|c|c|c|c|c|c|c|c|c|}
\hline \multirow{2}{*}{ 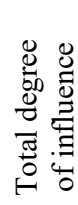 } & \multirow{2}{*}{ 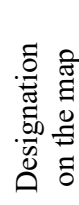 } & \multicolumn{10}{|c|}{ Type of load } & \multirow[b]{2}{*}{$I_{d}$} \\
\hline & & $k 1$ & $r 1$ & $k 2$ & $r 2$ & $k 3$ & $r 3$ & $k 4$ & $r 4$ & $k 5$ & $r 5$ & \\
\hline \multirow{2}{*}{$\Sigma \mathrm{S} 1$} & \multirow{2}{*}{ 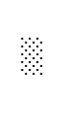 } & $0.2-0.7$ & \multirow{2}{*}{0.7} & 0 & \multirow{2}{*}{0.3} & $0.2-0.7$ & \multirow{2}{*}{0.5} & 0 & \multirow{2}{*}{0.4} & 1 & \multirow{2}{*}{0.3} & \multirow{2}{*}{$\leq 0.3$} \\
\hline & & 0.2 & & 1 & & 0.2 & & 0 & & 1 & & \\
\hline \multirow{6}{*}{$\Sigma \mathrm{S} 2$} & \multirow{6}{*}{ 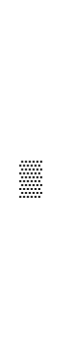 } & 0.2 & \multirow{6}{*}{0.7} & 0 & \multirow{6}{*}{0.3} & 0.2 & \multirow{6}{*}{0.5} & 1 & \multirow{6}{*}{0.4} & 1 & \multirow{6}{*}{0.3} & \multirow{6}{*}{$\begin{array}{l}>0.3 \\
\leq 0.5\end{array}$} \\
\hline & & 0.2 & & $1 / 0$ & & 0.5 & & $0 / 1$ & & 1 & & \\
\hline & & 0.5 & & $0 / 1$ & & 0.2 & & 0 & & $0 / 1$ & & \\
\hline & & 0.5 & & 0 & & 0.7 & & 0 & & 1 & & \\
\hline & & 0.7 & & 0 & & 0.5 & & 0 & & 1 & & \\
\hline & & 1 & & 0 & & 0.2 & & 0 & & 1 & & \\
\hline \multirow{6}{*}{$\Sigma \mathrm{S} 3$} & \multirow{6}{*}{ 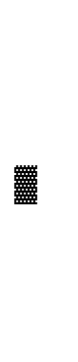 } & 0.2 & \multirow{6}{*}{0.7} & 1 & \multirow{6}{*}{0.3} & 0.2 & \multirow{6}{*}{0.5} & 1 & \multirow{6}{*}{0.4} & 1 & \multirow{6}{*}{0.3} & \multirow{6}{*}{$\begin{array}{l}>0.5 \\
\leq 0.7\end{array}$} \\
\hline & & 0.2 & & 1 & & 1 & & 0 & & 1 & & \\
\hline & & 0.5 & & $0 / 1$ & & $0.5-0.7$ & & $1 / 0$ & & 1 & & \\
\hline & & 0.7 & & $1 / 0$ & & 0.2 & & $0 / 1$ & & 1 & & \\
\hline & & 0.7 & & 0 & & 1 & & 0 & & 1 & & \\
\hline & & 1 & & 0 & & 0.5 & & 0 & & 1 & & \\
\hline \multirow{7}{*}{$\Sigma \mathrm{S} 4$} & & 0.2 & & 1 & & $0.5-1$ & & 1 & & 0 & & \\
\hline & & 0.5 & & 1 & & $0.2-0.5$ & & 1 & & 1 & & \\
\hline & & 0.5 & & $1 / 0$ & & $0.7-1$ & & $0 / 1$ & & 1 & & \\
\hline & $\mathbf{\square}$ & 0.7 & 0.7 & 1 & 0.3 & 0.2 & 0.5 & 1 & 0.4 & 1 & 0.3 & $>0.7$ \\
\hline & & 0.7 & & $0 / 1$ & & $0.5-1$ & & $1 / 0$ & & 1 & & \\
\hline & & 1 & & $1 / 0$ & & $0.2-0.5$ & & $0 / 1$ & & 1 & & \\
\hline & & 1 & & 0 & & $0.7-1$ & & 0 & & 1 & & \\
\hline & & $0.2-0.5$ & & 1 & & $0.7-1$ & & 1 & & 0 & & \\
\hline & & 0.7 & & 1 & & 0.2 & & 1 & & 0 & & \\
\hline$\Sigma \mathrm{S} 5$ & 0 & 0.7 & 0.7 & 1 & 0.3 & $0.5-1$ & 0.5 & 1 & 0.4 & 1 & 0.3 & $>1.0$ \\
\hline & & 1 & & 1 & & 0.2 & & 1 & & 0 & & \\
\hline & & 1 & & 1 & & $0.5-1$ & & 1 & & 1 & & \\
\hline
\end{tabular}




\section{Discussion}

The significance of the results obtained is that the developed methodology for constructing a damage map and the development of a prediction scale for conditions of exploitation made it possible to identify the relationship of problems in the natural ecosystems of the city and in the objects of urban development. Studies have shown that with the help of the developed cartographic model, causal relationships between the failure rate in anthropogenic systems of the city and the degree of ecosystem degradation can be traced. For example, such features of the territory, such as the absence of large roads near the location of the pipelines, the presence of lawns reduces the degree of load influence by even one or two orders, even if the groundwater is heavily polluted.

Also, based on the results of the work on the basis of so-called measures of similarity between sets of partial private evaluations, a classification of the city territory was carried out. As a result, five hazard classes of operating conditions were identified: zones of weak, medium, strong, maximum and extreme manifestation of environmental problems. During the quantitative assessment of each of the zones of manifestation of man-caused loads (Figure 4) it was determined that more than $3 / 5$ of the city's territory is a risk zone for the steel structures in the ground.

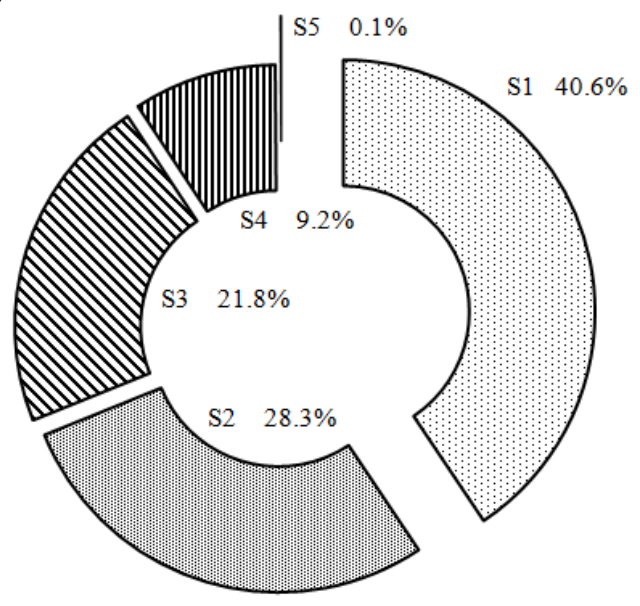

Fig. 4. The ratio of the city's areas with different levels of danger of conditions of exploitation.

It is also certain that the significance of the load $r$ for structures from different materials will differ. Therefore, the total influence index $I_{d}$ for them will also differ. However, the greatest impact on the attribution of conditions exploitation to a certain impact zone is provided by the quality index of the environment $k_{i}$.

It should also be noted that during the development of the cartogram, serious physicochemical studies were carried out to determine the influence of various loads of the urban environment on the durability of the objects. Because the:

$$
\sum_{i=1}^{n} r_{i}=f(\Delta t)=f\left\{\chi_{i}\right\} \neq 1 \quad \Rightarrow \quad \sum_{i=1}^{n} I_{d} \neq 1
$$

Therefore, the expert should be able tothe skill of assigning the $I_{d}$ intervals for each set of the degree of influence of $\Sigma \mathrm{S}_{\mathrm{i}}$, depending on the set of characteristics of the environment of exploitation $\{\chi i\}$ and their influence on the amount of time between failures $\Delta t$. In the course of further research, it is proposed to work on the refinement of these functional dependencies for the possibility of automating the calculation of $I_{d}$. 


\section{Conclusions}

Based on the studies carried out, the following conclusions can be drawn:

1. The existing system of emergency service map-case for engineering systems of the city on the basis of software complexes allows fixing the flow of failures and systematizing data on the technical state of engineering facilities. At the same time, there is no system for determining the correlation between the density distribution of the flow of failures in time and space and the magnitude of anthropogenic loads on the urban environment of exploitation.

2. Unlike engineering systems, data on the technical condition of buildings constructions are not systematized.. While the failure of load-bearing structures can lead to catastrophic consequences.

3. The algorithm proposed by the authors for the formation of a cartographic model can be used to predict and evaluate the risk of buildings constructions damage from environmental factors at the design, construction and repair stage.

4. The result of the implementation of the cartographic modeling system is the optimization of the monitoring system of the technical condition of the objects of townplanning activity and the quality of repair and construction works, as well as the correction of the type and timing of the implementation of preventive measures to restore the operability of the facilities.

\section{References}

1. E. Vialkova, M. Zemlyanova, A. Vorotnikova, D. Cherkashin, A. Voronov, L. Maksimov, MATEC Web of Conferences, 106, 07008 (2017)

2. URL: http://www.priroda.ru/lib/detail.php?ID $=5182$

3. URL: http://eco.tatarstan.ru/rus/gosdoklad-2016.htm

4. URL: http://centrnedra.ru/?Step=additional\&id=3

5. V. Azarov, N. Sergina, P. Sidyakin, I. Kovtunov, IOP Conf. Series: Earth and Environmental Science, 90, 012015 (2017)

6. V. Kucera, S. Fitz, Water, air and soil pollution, 1, 153-154 (1995)

7. E.V. Klimenko, L.A. Sheshukova, Eastern European Scientific Journal, 5, 102-104 (2014)

8. M. Dement'eva, IOP Conf. Series: Earth and Environmental Science, 90, 012052 (2017)

9. S.S.A. Kakhramanova, Vestnik of TSUAB, 1, 28-40 (2012)

10. O. Popova, T. Simankina, V. Lukinov, MATEC Web of Conferences, 106, 01013 (2017)

11. I.Yu. Vinokurov, I.N. Stepanov, Vladimir farmer, 1-2, 46-47 (2010)

12. Yu.M. Zinyukov, Proseedings Voronezh State University. Series: Geology, 1, 251-256 (2005)

13. L. Vlasova, G. Rakitina, S. Dolgov, S. Vasiliev, S. Stepanova, O. Krutkina, T. Fedorenko, Environment protection in oil and gas complex, 7, 38-47 (2013)

14. Ye.S. Utrobina, V.S. Pisarev, Interexpo Geo-Siberia, 2, 176-179 (2009)

15. O.N. Nicolaeva, Vestnik of the Siberian State University of Geosystems and Technologies (SSUGT), 4(32), 61-69 (2015)

16. A. Seidlová,Jakub Chromčák, MATEC Web of Conferences, 117, 00148 (2017) 
17. O. Popova, J. Glebova, A. Pustovgar, E3S Web of Conferences, 33, 02032 (2018)

18. E.E. Vasileva, V.S. Sherstnev, Modern problems of science and education, 3, 37 (2014)

19. I.A. Pukhlova, Y.V. Koroleva, M.A. Okhrimenko, V.A. Revunkov, International Baltic Maritime Forum, 4, 787-793 (2016)

20. URL: http://ecology-mef.narod.ru/geo/113.htm

21. URL: http://www.domrebenok.ru/blog/ekologicheskie-karty-moskvy-ipodmoskovya/?sphrase_id $=1218046$

22. URL: https://cdnimg.rg.ru/img/content/22/78/60/4660_1.gif

23. http://www.dpioos.ru/eco/ru/oopt 